\title{
Potential added value of a RT-qPCR method of SOX 11 expression, in the context of a multidisciplinary diagnostic assessment of $B$ cell malignancies
}

\author{
Julien Magne ${ }^{1}$, Alizée Jenvrin², Adrien Chauchet ${ }^{3}$, Olivier Casasnovas ${ }^{4}$, Anne Donzel ${ }^{1}$, Laurence Jego ${ }^{1}$, \\ Bernard Aral ${ }^{1}$, Julien Guy ${ }^{1}$, Nathalie Nadal ${ }^{1}$, Dewi Vernerey ${ }^{5}$, Patrick Callier ${ }^{1}$, Francine Garnache-Ottou ${ }^{2}$ \\ and Christophe Ferrand ${ }^{2,6^{*}}$ (B)
}

\begin{abstract}
Background: Expression of SRY [sex-determining region Y]-box11 (SOX11) is specific to mantle cell lymphoma (MCL) and contributes, in conjunction with immunoglobulin variable heavy chain gene mutation status, to the identification of two forms of this disease.

Methods: The aim of this report was firstly, to design an easy and suitable RT-qPCR method to quantify SOX11 mRNA expression in mantle cell lymphoma and other B cell malignancies with the proper reference gene; secondly, to define the best threshold of relative quantity of SOX 11 mRNA in order to reach the best compromise between sensitivity and specificity.

Results: For best discrimination of $M C L$ and non-MCL groups we determined an area under the curve (AUC) of 0.9750 and a threshold of 1.76 with $100 \%$ sensitivity and $88 \%$ specificity. AUC and threshold values of respectively $0.91 / 1.346$ [87\% sensitivity, 80\% specificity] and 0.9525/1.7120 [100\% sensitivity, 88\% specificity] for GAPDH and RPLP0 respectively denote that the RPLPO reference gene alone is sufficient for PCR housekeeping gene.
\end{abstract}

Conclusion: This work describes an RT-qPCR assay for SOX11 expression in order to better characterize MCL at diagnosis. Further studies on larger cohorts are needed to evaluate this molecular tool, especially for the follow-up of minimal residual disease.

Keywords: Mantle cell lymphoma, SOX11, RT-qPCR-reference genes, ROC curve analysis-threshold

\section{Introduction}

Mantle cell lymphoma (MCL) is a subtype of non-Hodgkin lymphoma that represents $5-10 \%$ of all lymphomas. It is considered to have a poor prognosis, with a high degree of clonal heterogeneity [1], leading to an overall 5 -year survival rate of $50-70 \%$ for limited or advanced stages respectively.

\footnotetext{
*Correspondence: christophe.ferrand@efs.sante.fr

${ }^{6}$ Laboratoire de Thérapeutique Immuno-Moléculaire et cellulaire des cancers, INSERM UMR1098, Etablissement Français du Sang Bourgogne/ Franche-Comté, 8, rue du Docteur Jean-François-Xavier Girod, 25020 Besançon, France

Full list of author information is available at the end of the article
}

If the $\mathrm{B}$ cell antigen receptor (BCR) plays a role in $\mathrm{MCL}$ lymphomagenesis and disease progression [2], most MCL harbor the $t(11 ; 14)(q 13 ; q 32)$ translocation resulting in overexpression of Cyclin D1 (CCND1) mRNA and transcription of the Cyclin D1 nuclear protein. However, transcriptomic studies [3] have revealed MCL cases without CCND1 dysregulation, suggesting that overexpression of CCND1 is not the only factor responsible for the disease. Independently of CCND1 expression, upregulation of SOX11 (SRY [sex-determining region-Y]-box 11), a neural transcription factor, has been detected in all cases of MCL, suggesting it has an important oncogenic role in the development of MCL tumor by regulating 
PAX5 [4]. SOX11, belonging to the family of genes $\mathrm{SOXC}$, is a transcript factor involved in the embryonic neurogenesis and tissue remodeling, also participating in the control of cell proliferation. It is known that SOX11 associated with WT1 has a synergic effect in the regulation of Wnt-4-promoted nephrogenesis. Several studies in solid tumors (i.e. breast cancer [5]) demonstrate that SOX11, through the expression modulation by epigenetics regulation, plays a transcriptional role that promotes growth and cell differentiation [6]. SOX11 directly binds also to the regulatory regions of PAX5 and BCL6, 2 crucial transcription factors involved in early B-cell development and late differentiation (PAX5) [7].

Absence of SOX11 over-expression in lymphoid progenitor or in mature normal B-cells, correlated with IGHV mutational status in MCL, makes SOX11 a potential biomarker for CCND1-negative MCL, both at diagnosis and in the evaluation of minimal residual disease (MRD). Although the functional role of SOX11 and its prognostic impact on overall survival are still debated, the 2016 revision of the World Health Organization (WHO) classification of lymphoid neoplasms [8] now differentiates two MCL subtypes, with different clinicpathological manifestations and molecular pathways. The first subtype, with mainly unmutated/minimally mutated IGHV and mostly SOX11-positive, is associated with leukemic non-nodal MCL with bone marrow, splenic and peripheral blood involvement. The second subtype is mostly SOX11-negative, is associated with mutated IGHV and generally indolent, although it may become aggressive by acquiring TP 53 mutation.

For diagnosis purpose, SOX11 expression is primarily analyzed by immunohistochemistry (IHC) or flow cytometry [9]. However, although the availability of the high specificity monoclonal antibody MRQ-58 has improve the diagnosis analysis, the lack of defined cutoff levels of SOX11 expression may generate conflicting results. Thus, molecular biology SOX11 quantification [10], may help to solve and overcome these difficulties but may also be used for MRD quantification.

We aimed to study SOX11 expression with an easy and suitable RT-qPCR method, using appropriate housekeeping genes, and to apply this assay to a cohort of B-cell malignancies, in order to define an easy-to-use molecular tool for distinguishing MCL from other B lymphoproliferative disorders.

\section{Materials and methods}

62 adult patients with $(n=60)$ or without $(n=2)$ hematological malignancies were included in the study. Samples were collected from November 2012 to January 2016 at the onco-hematology laboratories of the University Hospitals of Dijon and Besançon, France. 51 samples were collected from routine diagnosis based on semi-quantitative CCND1 expression analysis. MCL diagnosis was established by multidisciplinary analysis, including clinical and paraclinical data, imagery, cytology, pathology, cytogenetics and molecular biology. Among these 54 samples, 31 had CCND1 overexpression, divided between aggressive $(\mathrm{cMCL}, \mathrm{n}=21)$, indolent MCL (iMCL, $n=9$ ) and Multiple Myelom (MM, $\mathrm{n}=1$ ), whereas the other 23 had Marginal Zone Lymphomas (MZL, $\mathrm{n}=4$ ), Waldenström's Macroglobulinemia (WM, $n=2)$, Chronic lymphocytic leukemia and small lymphocytic lymphoma (CLL/SLL, $n=4)$, Follicular lymphoma ( $\mathrm{FL}, \mathrm{n}=1)$, diffuse large $\mathrm{B}$ cell lymphoma (DLBCL, $\mathrm{n}=1$ ), (iMCL, $\mathrm{n}=3$ ), (cMCL, $\mathrm{n}=1$ ), and also RNA samples from patients in complete remission (CR, $\mathrm{n}=5$ ) of their hematological malignancies, previously CCND1 positive. The other eight samples were obtained from Chronic Lymphoid Leukemia (CLL, $\mathrm{n}=3$ ), Acute Lymphoid Leukemia (ALL, $\mathrm{n}=3$ ) or controls $(\mathrm{n}=2)$ (Fig. 1). All samples were obtained after patients provided informed consent, and the study was approved by the local institutional review board.

Mononuclear cells were isolated from the EDTA harvesting tube by Ficoll density Centrifugation. RNAs were extracted using either TRIZOL reagent or RNeasy Mini Kit (Qiagen, Courtaboeuf, France) DNAse exposure was added in order to avoid gDNA contamination. Reverse transcription was performed from RNA. Real time PCR quantification was done in triplicate, in a $20 \mu \mathrm{l}$ reaction final volume, from $2 \mu \mathrm{l}$ of cDNA, in a CFX96 thermocycler (Biorad, Marne La Coquette, France) using exonic primer pairs PrimePCR ${ }^{\mathrm{TM}}$ PreAmp, Biorad) designed for $\mathrm{SYBR}^{\circledR}$ Green gene expression analysis of target SOX11 (Ref \#qHsaCED0018676) or housekeeping genes ribosomal protein, large, P0 (RPLP0, ref\#qHsaCED0038653) or glyceraldehyde-3-phosphate dehydrogenase (GAPDH, ref \#qHsaCED0038674). To improve specificity, a PCR mix containing a Sso7d fusion DNA polymerase (Biorad) was used. Standard two step PCR, according to the manufacturer's recommendations, was run in a CFX96 thermal cycler (Biorad).

Results were collected and analyzed using the CFX Manager Software (Biorad). Gene expression was assessed using relative quantification with the $\Delta \Delta C T$ method [11] taking into consideration that qPCR efficiencies are equivalent between target and housekeeping gene PCR. Results were retained if at least two replicates had a difference of $<0.4 \mathrm{Ct}$. PCR efficiencies were assessed by serial log dilutions of a cDNA synthetized from an MCL diagnosis sample in order to generate a standard curve of Ct. Relative expression quantification was calculated against a normal control (calibrator) obtained from either peripheral blood mononuclear cells, 


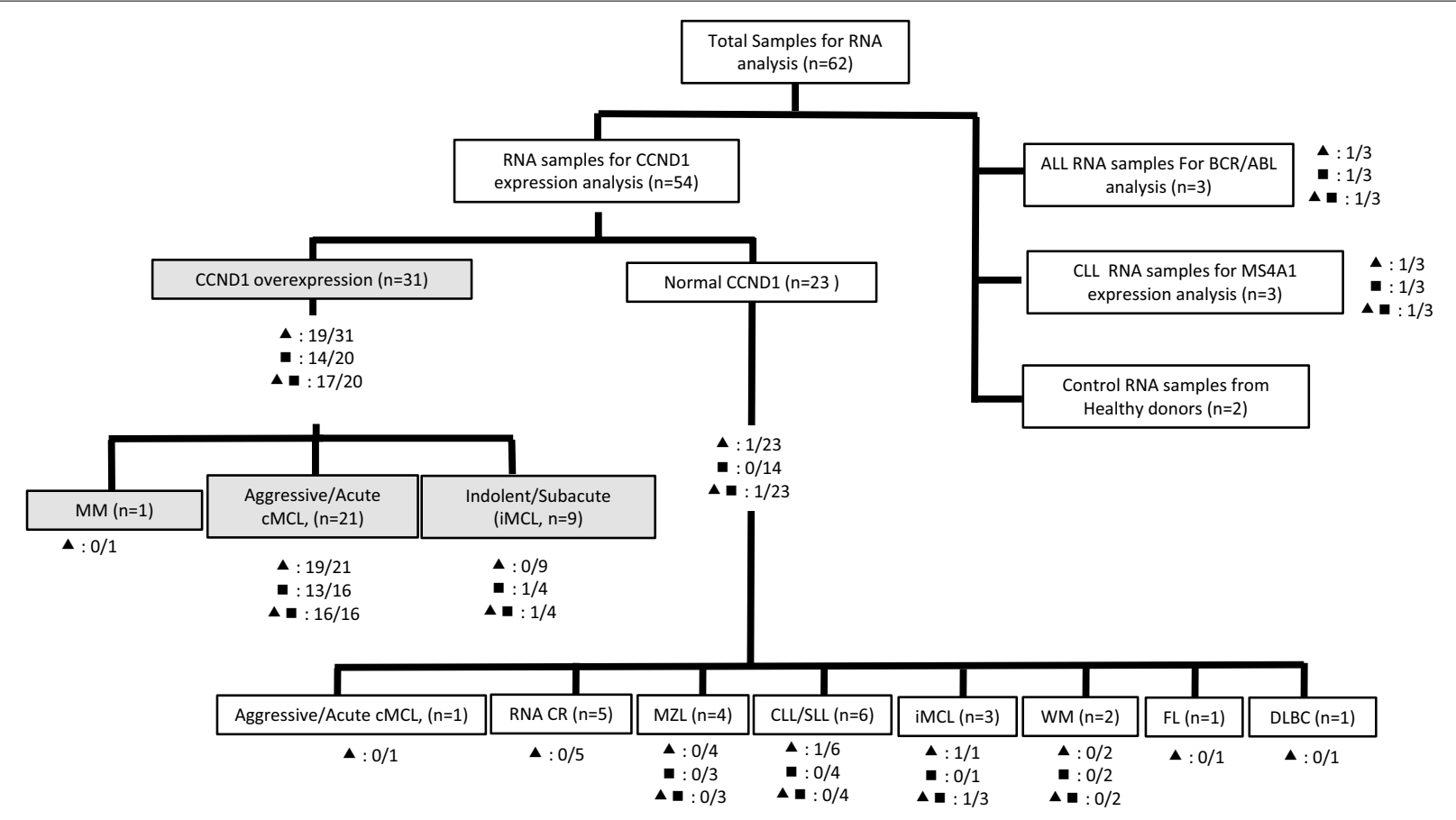

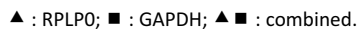

Fig. 1 Samples tree screened with SOX11 RTqPCR. Number of patients positive for SOX 11 expression respectively with the use of (filled triangle) RPLP0, (filled square) GAPDH or both (filled triangle, filled square) reference genes. Grey boxes represent CCND1 positive samples; CR Complete remission

or B lymphocyte isolation from healthy donors $(\mathrm{n}=3)$, according to either individual or associated housekeeping genes.

Statistical analyses were performed using $\mathrm{R}$ software version 2.15.2 (R Development Core Team; http://www.rproject.org). A p $<0.05$ was considered statistically significant and all tests were two-sided.

\section{Results}

The qPCR efficiencies were calculated from the slope of the regression line, plotted from PCR results obtained from Log10 serial dilutions of the same MCL sample. Efficiencies of 98, 103, and 109\% for respectively SOX11, GAPDH and RPLP0 PCR were similar according the MiQE guidelines [12] (Additional file 1: Figure S1). For the calibrator $(n=2)$, an average $C t$ of 32.6 was obtained for the target SOX11 PCR, whereas we found Ct means of 19.8 and 21.1 respectively for control RPLP0 or GAPDH PCR.

We analyzed typical CCND1 positive MCL samples. For this group, we found a level of relative fold increase (RFI) SOX11 expression of 3352 [min-max: 27-9587.3], 763 [5-4015] or 1070 times [24-2953] higher than within the calibrator, according to RPLP0, GAPDH or both reference genes respectively. Remarkably, one patient with a B-ALL showed a level of SOX 11 expression at a level equal to 209 [0.1-209] (GAPDH), 358 [2-358] (RPLP0), and 273 [0.4-273] (GAPDH \& RPLP0) times the control. Interestingly, an IGVH-mutated-CLL patient also had a high level of SOX11 compared to the calibrator: 80 [1-80], 66 [0.2-66], and 72 [0.4-72] respectively for GAPDH, RPLP0 and both housekeeping genes.

Interestingly, all 5 RNA samples harvested from patients in CR of their hematological malignancies and CCND1 positive at diagnosis were categorized at low level of SOX11 expression.

Log rq of RFI value was plotted for MCL or "non-MCL" samples for isolated or combined reference genes (Fig. 2). For every reference gene, the difference was highly significant ( $\mathrm{p}<0.001$ ). Unconditional logistic regression was then performed to model the MCL probability according to the level of log rq gene expressions. A receiver operating characteristic (ROC) curve was constructed (Additional file 1: Figure S2), with calculation of the area under the curve (AUC). The Youden index, representing the difference between the true positive rate and the false positive rate, was maximized to obtain the optimal threshold log rq gene expressions value for the discrimination of MCL and non-MCL groups. 


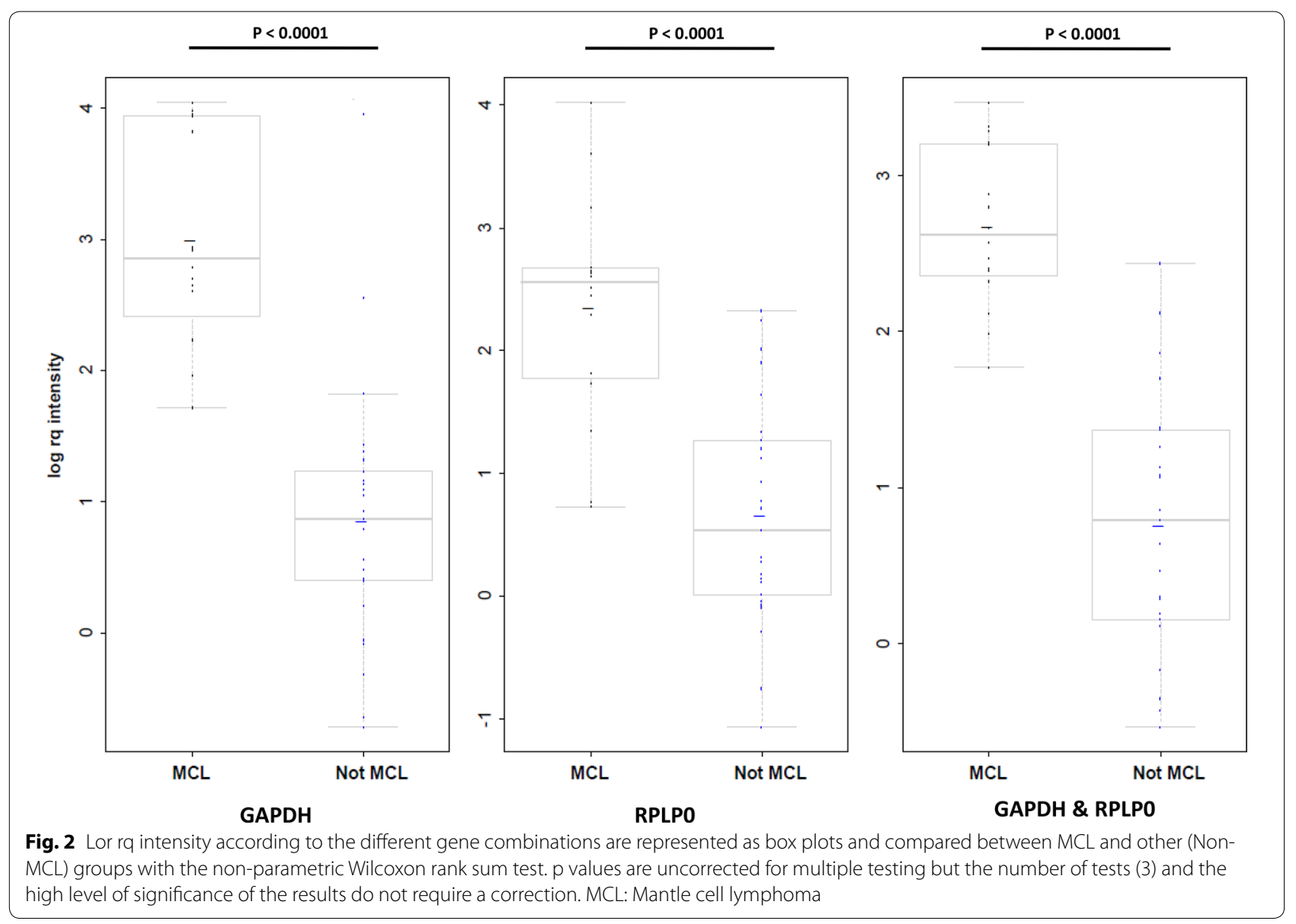

For both reference genes, we determined an AUC of 0.9750 and a threshold of 1.76 with $100 \%$ sensitivity and $88 \%$ specificity. AUC and threshold values of respectively 0.91/1.346 (87\% sensitivity, 80\% specificity) and 0.9476/1.7120 [100\% sensitivity, 88\% specificity] for GAPDH and RPLP0 respectively denote that the RPLP0 reference gene alone is suffcient for PCR housekeeping gene.

\section{Discussion/conclusion}

We describe here a simple RT-qPCR protocol that is easy to carry out, to evaluate relative SOX11 expression, and the discriminatory potential of the assay for the diagnosis of MCL disease. We evaluated two different housekeeping genes, either isolated or combined, according to qPCR efficiencies (slopes), in order to apply the $\triangle \triangle C T$ method. As demonstrated, the use of both reference genes GAPDH and RPLP0 allows better discrimination of MCL versus non-MCL samples. However, statistical ROC curve analysis showed that only RPLP0 is required, and is sufficient alone. This will simplify the molecular biology assay and also reduce costs. We also define the best threshold to discriminate MCL versus non-MCL, with a sensitivity of $100 \%$ a specificity of $88 \%$.
Despite the low number of samples in our cohort, we detected high level expression in a CLL case. This is in agreement with literature reports of SOX11 expression in $35 \%$ of CLL, whereas the remaining 65\% lack expression, in association with the IGVH mutational status and aggressiveness of disease [13]. We observed a high level of SOX11 expression in all 3 ALL samples, which has also been reported in the literature for ALL Phi negative case or cell lines. As previously reported by Vegliante et al. [14], SOX11 expression is associated with unmethylated DNA and presence of activating histone marks (H3K9/14Ac and H3K4me3) in some aggressive B-cell neoplasms, whereas it is methylated (with silent histones H3K9me2and H3K27me3) in adult stem cells, normal hematopoietic cells and other lymphoid neoplasms.

In addition to CCND1 expression by RTqPCR, $\mathrm{t}(11 ; 14)$ chromosome analysis by FISH or BCL1/JH clonotypic DNA rearrangements by PCR, another method could be useful both to define the disease at diagnosis, for stratification and prognostic value, but also for MRD follow-up. Indeed, MRD quantification is known to be an indicator of treatment response in MCL to prevent clinical relapse [15]. Interestingly, SOX11 expression of RNA samples 
from patients previously CCND1 positive, are negative at time of CR. Ideally, a multiplex RTqPCR of both CCND1 and SOX11 with 2 appropriate reference genes may enable improved molecular characterization of MCL and follow-up of MRD, for example in order to monitor ibrutinib or lenalidomide treatment efficacy [16]. Association with quantification of CD20 alternative transcripts [17] may be useful, although this molecular tool evaluation has to be properly tested in support of a clinical trial.

For diagnostic purposes, the use of the mantle cell lymphoma international prognostic index (MIPI) in routine clinical practice has shown some limitations, because it imperfectly separates low and intermediate risk groups [18]. Thus, molecular RT-qPCR quantification of SOX11 should add prognostic information to the MIPI, and make it possible to better define patients at risk of poorer outcome who may eligible for alternative treatments, thus minimizing treatment-related morbidity.

In conclusion, this work describes an RT-qPCR assay for SOX11 expression in order to better characterize $\mathrm{MCL}$ at diagnosis. Further studies on larger cohorts are needed to evaluate this molecular tool, especially for the follow-up of MRD.

\section{Additional file}

Additional file 1. Figures.

\begin{abstract}
Abbreviations
SOX11: SRY [sex-determining region Y]-box11; RT-qPCR: reverse transcription quantitative polymerase chain reaction; MCL: mantle cell lymphoma; FL: follicular lymphoma; $\mathrm{MM}$ : multiple myeloma; $\mathrm{DLBCL}$ : diffuse large $\mathrm{B}$ cell lymphoma; CCND1: Cyclin D1; MRD: minimal residual disease; WHO: World Health Organization; IGHV: immunoglobulin heavy chain variable; IHC: immunohistochemistry; MZL: marginal zone lymphomas; WM: Waldenström's Macroglobulinemia; CLL: chronic lymphocytic leukemia; SLL: small lymphocytic lymphoma; ALL: acute lymphoid leukemia; RPLPO: housekeeping genes ribosomal protein large P0; GAPDH: glyceraldehyde-3-phosphate dehydrogenase; cDNA: complementary DNA; gDNA: genomic DNA; ROC: receiver operating characteristic; AUC: area under the curve; MIPI: mantle cell lymphoma international prognostic index; CR: complete remission.
\end{abstract}

\section{Authors' contributions}

JM perform the experiments and write the original draft; AJ contribute to the writing of manuscript; AC, OC, FGO, JG provide clinical samples from patients; AD, PC help in molecular analysis; DW perform statistical analysis; CF: Design the study, analyze the data and write the manuscript. All authors read and approved the final manuscript

\section{Author details}

${ }^{1}$ INSERM, UMR866, Faculté des Sciences de Santé, Univ. Bourgogne FrancheComté, 21000 Dijon, France. ${ }^{2}$ INSERM, EFS BFC, UMR1098, Univ. Bourgogne Franche-Comté, Besançon 25000, France. ${ }^{3}$ Department of Hematology, University Hospital of Besancon, 25000 Besançon, France. ${ }^{4}$ Department of Hematology, University Hospital of Dijon, 21000 Dijon, France. ${ }^{5}$ Univ. Bourgogne Franche-Comté, EA 3181, 25000 Besançon, France. ${ }^{6}$ Laboratoire de Thérapeutique Immuno-Moléculaire et cellulaire des cancers, INSERM UMR1098, Etablissement Français du Sang Bourgogne/Franche-Comté, 8, rue du Docteur Jean-François-Xavier Girod, 25020 Besançon, France.

\section{Acknowledgements}

Thanks to Sarah Odrion for reading the manuscript and to technicians of the Onco-Hematology Lab of the EFSBFC for technical assistance.

\section{Competing interests}

The authors declare that they have no competing interests.

\section{Availability of data and materials}

Raw datasets used during the current study are stored and available from the corresponding author on reasonable request.

\section{Consent for publication}

Not applicable.

\section{Ethics approval and consent to participate}

All samples were obtained after patients provided informed consent, and the study was approved by the local institutional review board.

\section{Funding}

Not applicable.

\section{Publisher's Note}

Springer Nature remains neutral with regard to jurisdictional claims in published maps and institutional affiliations.

Received: 14 November 2017 Accepted: 2 February 2018

Published online: 20 February 2018

References

1. Cheah CY, Seymour JF, Wang ML. Mantle cell lymphoma. J Clin Oncol. 2016;34(11):1256-69.

2. Fichtner $\mathrm{M}$, et al. The role of $\mathrm{B}$ cell antigen receptors in mantle cell lymphoma. J Hematol Oncol. 2017;10(1):164.

3. Fu K, et al. Cyclin D1-negative mantle cell lymphoma: a clinicopathologic study based on gene expression profiling. Blood. 2005;106(13):4315-21.

4. Vegliante MC, et al. SOX11 regulates PAX5 expression and blocks terminal B-cell differentiation in aggressive mantle cell lymphoma. Blood. 2013;121(12):2175-85.

5. Shepherd $\mathrm{JH}$, et al. The SOX 11 transcription factor is a critical regulator of basal-like breast cancer growth, invasion, and basal-like gene expression. Oncotarget. 2016;7(11):13106-21.

6. LuTX, Li JY, Xu W. The role of SOX11 in mantle cell lymphoma. Leuk Res. 2013;37(11):1412-9.

7. Bea S, Amador V. Role of SOX 11 and genetic events cooperating with Cyclin D1 in mantle cell lymphoma. Curr Oncol Rep. 2017;19(6):43.

8. Swerdlow SH, et al. The 2016 revision of the World Health Organization classification of lymphoid neoplasms. Blood. 2016;127(20):2375-90.

9. Wasik AM, et al. Flow cytometric analysis of SOX11: a new diagnostic method for distinguishing B-cell chronic lymphocytic leukemia/small lymphocytic lymphoma from mantle cell lymphoma. Leuk Lymphoma. 2015;56(5):1425-31.

10. Lord $M$, et al. The utility of mRNA analysis in defining SOX 11 expression levels in mantle cell lymphoma and reactive lymph nodes. Haematologica. 2015;100(9):e369-72.

11. Pfaffl MW. A new mathematical model for relative quantification in realtime RT-PCR. Nucleic Acids Res. 2001;29(9):e45.

12. Bustin SA, et al. The MIQE guidelines: minimum information for publication of quantitative real-time PCR experiments. Clin Chem. 2009;55(4):611-22.

13. Roisman A, et al. SOX11 expression in chronic lymphocytic leukemia correlates with adverse prognostic markers. Tumour Biol. 2015;36(6):4433-40.

14. Vegliante MC, et al. Epigenetic activation of SOX11 in lymphoid neoplasms by histone modifications. PLOS ONE. 2011;6(6):e21382.

15. Andersen NS, et al. Pre-emptive treatment with rituximab of molecular relapse after autologous stem cell transplantation in mantle cell lymphoma. J Clin Oncol. 2009;27(26):4365-70. 
16. Wang $M$, et al. Observational study of lenalidomide in patients with mantle cell lymphoma who relapsed/progressed after or were refractory/ intolerant to ibrutinib (MCL-004). J Hematol Oncol. 2017;10(1):171.

17. Gamonet C, et al. New CD20 alternative splice variants: molecular identification and differential expression within hematological B cell malignancies. Exp Hematol Oncol. 2015;5:7.
18. Nordstrom L, et al. SOX11 and TP53 add prognostic information to MIPI in a homogenously treated cohort of mantle cell lymphoma-a nordic lymphoma group study. Br J Haematol. 2014;166(1):98-108.
Submit your next manuscript to BioMed Central and we will help you at every step:

- We accept pre-submission inquiries

- Our selector tool helps you to find the most relevant journal

- We provide round the clock customer support

- Convenient online submission

- Thorough peer review

- Inclusion in PubMed and all major indexing services

- Maximum visibility for your research

Submit your manuscript at www.biomedcentral.com/submit
() Biomed Central 